\title{
WEAK BRAIDED BIALGEBRAS AND WEAK ENTWINING STRUCTURES
}

\author{
J. N. ALONSO ÁLVAREZ, J. M. FERNÁNDEZ VILABOA and \\ R. GONZÁLEZ RODRÍGUEZ ${ }^{凶}$
}

(Received 11 December 2008)

\begin{abstract}
In this paper we clarify and improve the notion of weak braided bialgebra using weak entwining structures. As a main result we show that the notion of weak braided bialgebra can be rewritten in terms of weak entwining structures.
\end{abstract}

2000 Mathematics subject classification: primary 18D10; secondary 16W30.

Keywords and phrases: monoidal category, weak braided hopf algebra, entwining structures.

\section{Introduction}

Weak braided bialgebras and weak braided Hopf algebras were defined by us in [1] as a generalization to the braided setting of the notions of weak bialgebra and weak Hopf algebra introduced by Böhm et al. [4]. The definition of these weak braided structures was inspired by the work of Takeuchi [11] and motivated by the study and characterization of weak Hopf algebra projections that we finished in [1] where, for a weak Hopf algebra $H$ with invertible antipode, we obtain a one-to-one correspondence between Hopf algebras in the category of left-left Yetter-Drinfeld modules over $H$ and pairs of morphisms of weak Hopf algebras $f: H \rightarrow B, g: B \rightarrow H$ such that $g \circ f=\mathrm{id}_{H}$. In order to establish this result, which generalizes the classical theorems proved by Radford [10] and Majid [8] to the weak setting, we had defined previously the notion of weak Yang-Baxter operators and had showed that it is possible to construct examples of these weak operators working with the category of YetterDrinfeld modules over a weak Hopf algebra with invertible antipode.

Roughly speaking, a weak braided bialgebra $D$ in a strict monoidal category is an algebra-coalgebra with a weak Yang-Baxter operator $t_{D, D}: D \otimes D \rightarrow D \otimes D$, satisfying some compatibility conditions. This definition generalizes the one introduced by Takeuchi in [11], that is, the definition of braided bialgebra, and 
the classical notion of bialgebra in a braided or symmetric category. Moreover, as particular instances, we recover the definition of weak bialgebra and, if the weak Yang-Baxter operator is the braiding of a braided category, we formulate the new notion of weak bialgebra in a braided monoidal setting.

The aim of this paper is to improve the definition of weak braided bialgebra introduced in [1] and discuss how working with these algebraic objects it is possible to obtain examples of weak entwining structures (see [5] for the definition). Moreover, we prove that weak entwining structures provide a good characterization of weak braided bialgebras; that is, in the final theorem of this paper we prove that the definition of weak braided bialgebra can be stated in terms of weak entwining structures.

\section{Relations between weak braided Hopf algebras and entwining structures}

We assume that the reader is familiar with the machinery of monoidal categories. Throughout the paper we work in a strict monoidal category $(\mathcal{C}, \otimes, K)$ where every idempotent splits; that is, for every morphism $q: Y \rightarrow Y$ such that $q=q \circ q$, then there exist an object $Z$ and morphisms $i: Z \rightarrow Y$ and $p: Y \rightarrow Z$ satisfying $q=i \circ p$ and $p \circ i=\mathrm{id}_{Z}$ where $\mathrm{id}_{Z}$ denotes the identity morphism in $Z$. For simplicity of notation, given objects $M, N, P$ in $\mathcal{C}$ and a morphism $f: M \rightarrow N$, we write $P \otimes f$ for $\operatorname{id}_{P} \otimes f$ and $f \otimes P$ for $f \otimes \operatorname{id}_{P}$.

An algebra in $\mathcal{C}$ is a triple $A=\left(A, \eta_{A}, \mu_{A}\right)$ where $A$ is an object in $\mathcal{C}$ and $\eta_{A}: K \rightarrow A$ (unit), $\mu_{A}: A \otimes A \rightarrow A$ (product) are morphisms in $\mathcal{C}$ such that $\mu_{A} \circ$ $\left(A \otimes \eta_{A}\right)=\mathrm{id}_{A}=\mu_{A} \circ\left(\eta_{A} \otimes A\right), \mu_{A} \circ\left(A \otimes \mu_{A}\right)=\mu_{A} \circ\left(\mu_{A} \otimes A\right)$. A coalgebra in $\mathcal{C}$ is a triple $D=\left(D, \varepsilon_{D}, \delta_{D}\right)$ where $D$ is an object in $\mathcal{C}$ and $\varepsilon_{D}: D \rightarrow K$ (counit), $\delta_{D}: D \rightarrow D \otimes D$ (coproduct) are morphisms in $\mathcal{C}$ such that $\left(\varepsilon_{D} \otimes D\right) \circ \delta_{D}=\mathrm{id}_{D}=$ $\left(D \otimes \varepsilon_{D}\right) \circ \delta_{D},\left(\delta_{D} \otimes D\right) \circ \delta_{D}=\left(D \otimes \delta_{D}\right) \circ \delta_{D}$.

If $A$ is an algebra, $B$ is a coalgebra and $\alpha: B \rightarrow A, \beta: B \rightarrow A$ are morphisms, we define the convolution product by $\alpha \wedge \beta=\mu_{A} \circ(\alpha \otimes \beta) \circ \delta_{B}$.

Let $D \in \mathcal{C}$ and let $t_{D, D}: D \otimes D \rightarrow D \otimes D$ be a morphism in $\mathcal{C}$. We will say that $t_{D, D}$ satisfies the Yang-Baxter equation if

$$
\left(t_{D, D} \otimes D\right) \circ\left(D \otimes t_{D, D}\right) \circ\left(t_{D, D} \otimes D\right)=\left(D \otimes t_{D, D}\right) \circ\left(t_{D, D} \otimes D\right) \circ\left(D \otimes t_{D, D}\right) .
$$

The notion of weak Yang-Baxter operator is a generalization of the classical definition of Yang-Baxter operator and was introduced by the authors in [1]. The definition is the following.

Definition 2.1. Let $D \in \mathcal{C}$. A weak Yang-Baxter operator is a morphism $t_{D, D}$ : $D \otimes D \rightarrow D \otimes D$ in $\mathcal{C}$ satisfying the following conditions.

(a1) The morphism $t_{D, D}$ satisfies the Yang-Baxter equation.

(a2) There exists an idempotent morphism $\nabla_{D \otimes D}: D \otimes D \rightarrow D \otimes D$ such that:

$(\mathrm{a} 2-1)\left(\nabla_{D \otimes D} \otimes D\right) \circ\left(D \otimes \nabla_{D \otimes D}\right)=\left(D \otimes \nabla_{D \otimes D}\right) \circ\left(\nabla_{D \otimes D} \otimes D\right)$;

$(\mathrm{a} 2-2)\left(\nabla_{D \otimes D} \otimes D\right) \circ\left(D \otimes t_{D, D}\right)=\left(D \otimes t_{D, D}\right) \circ\left(\nabla_{D \otimes D} \otimes D\right)$;

$(\mathrm{a} 2-3)\left(t_{D, D} \otimes D\right) \circ\left(D \otimes \nabla_{D \otimes D}\right)=\left(D \otimes \nabla_{D \otimes D}\right) \circ\left(t_{D, D} \otimes D\right)$;

(a2-4) $t_{D, D} \circ \nabla_{D \otimes D}=\nabla_{D \otimes D} \circ t_{D, D}=t_{D, D}$. 
(a3) There exists a morphism $t_{D, D}^{\prime}: D \otimes D \rightarrow D \otimes D$ such that:

(a3-1) $t_{D, D}^{\prime}$ satisfies the Yang-Baxter equation;

(a3-2) the morphism $p_{D, D} \circ t_{D, D} \circ i_{D, D}: D \times D \rightarrow D \times D$ is an isomorphism with inverse $p_{D, D} \circ t_{D, D}^{\prime} \circ i_{D, D}: D \times D \rightarrow D \times D$, where $p_{D, D}$ and $i_{D, D}$ are the morphisms such that $i_{D, D} \circ p_{D, D}=\nabla_{D \otimes D}$ and $p_{D, D} \circ$ $i_{D, D}=\operatorname{id}_{D \times D}$ being $D \times D$ the image of $\nabla_{D \otimes D}$;

(a3-3) $t_{D, D}^{\prime} \circ \nabla_{D \otimes D}=\nabla_{D \otimes D} \circ t_{D, D}^{\prime}=t_{D, D}^{\prime}$.

Note that if $\nabla_{D \otimes D}=\mathrm{id}_{D \otimes D}$ the morphism $t_{D, D}$ is an isomorphism and we have the usual definition of Yang-Baxter operator in the sense of Joyal and Street [6]. Also, as a direct consequence of this definition, the idempotent morphism $\nabla_{D \otimes D}$ satisfies the Yang-Baxter equation and we have that $t_{D, D}$ is a weak Yang-Baxter operator if and only if $t_{D, D}^{\prime}$ is a weak Yang-Baxter operator. Also, by [1, Proposition 1.3], we have $t_{D, D}^{\prime} \circ t_{D, D}=t_{D, D} \circ t_{D, D}^{\prime}=\nabla_{D \otimes D}$.

Now we recall the definition of weak braided bialgebra introduced in [1].

DEFINITION 2.2. A weak braided bialgebra $D$ is an object in $\mathcal{C}$ with an algebra structure $\left(D, \eta_{D}, \mu_{D}\right)$ and a coalgebra structure $\left(D, \varepsilon_{D}, \delta_{D}\right)$ such that there exists a weak Yang-Baxter operator $t_{D, D}: D \otimes D \rightarrow D \otimes D$ with associated idempotent $\nabla_{D \otimes D}$ satisfying the following conditions.

(b1) We have:

(b1-1) $\mu_{D} \circ \nabla_{D \otimes D}=\mu_{D}$;

(b1-2) $\nabla_{D \otimes D} \circ\left(\mu_{D} \otimes D\right)=\left(\mu_{D} \otimes D\right) \circ\left(D \otimes \nabla_{D \otimes D}\right)$;

(b1-3) $\nabla_{D \otimes D} \circ\left(D \otimes \mu_{D}\right)=\left(D \otimes \mu_{D}\right) \circ\left(\nabla_{D \otimes D} \otimes D\right)$.

(b2) We have:

(b2-1) $\nabla_{D \otimes D} \circ \delta_{D}=\delta_{D}$;

(b2-2) $\left(\delta_{D} \otimes D\right) \circ \nabla_{D \otimes D}=\left(D \otimes \nabla_{D \otimes D}\right) \circ\left(\delta_{D} \otimes D\right)$;

(b2-3) $\left(D \otimes \delta_{D}\right) \circ \nabla_{D \otimes D}=\left(\nabla_{D \otimes D} \otimes D\right) \circ\left(D \otimes \delta_{D}\right)$.

(b3) The morphisms $\eta_{D}, \mu_{D}, \varepsilon_{D}$ and $\delta_{D}$ commute with $t_{D, D}$, that is:

(b3-1) $t_{D, D} \circ\left(\eta_{D} \otimes D\right)=\nabla_{D \otimes D} \circ\left(D \otimes \eta_{D}\right)$;

(b3-2) $t_{D, D} \circ\left(D \otimes \eta_{D}\right)=\nabla_{D \otimes D} \circ\left(\eta_{D} \otimes D\right)$;

(b3-3) $t_{D, D} \circ\left(\mu_{D} \otimes D\right)=\left(D \otimes \mu_{D}\right) \circ\left(t_{D, D} \otimes D\right) \circ\left(D \otimes t_{D, D}\right)$;

(b3-4) $t_{D, D} \circ\left(D \otimes \mu_{D}\right)=\left(\mu_{D} \otimes D\right) \circ\left(D \otimes t_{D, D}\right) \circ\left(t_{D, D} \otimes D\right)$;

$(\mathrm{b} 3-5)\left(\varepsilon_{D} \otimes D\right) \circ t_{D, D}=\left(D \otimes \varepsilon_{D}\right) \circ \nabla_{D \otimes D}$;

(b3-6) $\left(D \otimes \varepsilon_{D}\right) \circ t_{D, D}=\left(\varepsilon_{D} \otimes D\right) \circ \nabla_{D \otimes D}$;

(b3-7) $\left(\delta_{D} \otimes D\right) \circ t_{D, D}=\left(D \otimes t_{D, D}\right) \circ\left(t_{D, D} \otimes D\right) \circ\left(D \otimes \delta_{D}\right)$;

(b3-8) $\left(D \otimes \delta_{D}\right) \circ t_{D, D}=\left(t_{D, D} \otimes D\right) \circ\left(D \otimes t_{D, D}\right) \circ\left(\delta_{D} \otimes D\right)$.

(b4) $\delta_{D} \circ \mu_{D}=\left(\mu_{D} \otimes \mu_{D}\right) \circ\left(D \otimes t_{D, D} \otimes D\right) \circ\left(\delta_{D} \otimes \delta_{D}\right)$.

(b5) $\varepsilon_{D} \circ \mu_{D} \circ\left(\mu_{D} \otimes D\right)=\left(\left(\varepsilon_{D} \circ \mu_{D}\right) \otimes\left(\varepsilon_{D} \circ \mu_{D}\right)\right) \circ\left(D \otimes \delta_{D} \otimes D\right)$

$=\left(\left(\varepsilon_{D} \circ \mu_{D}\right) \otimes\left(\varepsilon_{D} \circ \mu_{D}\right)\right) \circ\left(D \otimes\left(t_{D, D}^{\prime} \circ \delta_{D}\right) \otimes D\right)$.

(b6) $\left(\delta_{D} \otimes D\right) \circ \delta_{D} \circ \eta_{D}=\left(D \otimes \mu_{D} \otimes D\right) \circ\left(\left(\delta_{D} \circ \eta_{D}\right) \otimes\left(\delta_{D} \circ \eta_{D}\right)\right)$

$=\left(D \otimes\left(\mu_{D} \circ t_{D, D}^{\prime}\right) \otimes D\right) \circ\left(\left(\delta_{D} \circ \eta_{D}\right) \otimes\left(\delta_{D} \circ \eta_{D}\right)\right)$. 
The weak braided bialgebra $D$ is called a weak braided Hopf algebra in $\mathcal{C}$ when we have the following property.

(b7) There exists a morphism $\lambda_{D}: D \rightarrow D$ in $\mathcal{C}$ (called the antipode of $D$ ) satisfying:

$(\mathrm{b} 7-1) \operatorname{id}_{D} \wedge \lambda_{D}=\left(\left(\varepsilon_{D} \circ \mu_{D}\right) \otimes D\right) \circ\left(D \otimes t_{D, D}\right) \circ\left(\left(\delta_{D} \circ \eta_{D}\right) \otimes D\right)$;

$(\mathrm{b} 7-2) \lambda_{D} \wedge \mathrm{id}_{D}=\left(D \otimes\left(\varepsilon_{D} \circ \mu_{D}\right)\right) \circ\left(t_{D, D} \otimes D\right) \circ\left(D \otimes\left(\delta_{D} \circ \eta_{D}\right)\right)$;

(b7-3) $\lambda_{D} \wedge \mathrm{id}_{D} \wedge \lambda_{D}=\lambda_{D}$.

Note that, if $D$ is a weak braided bialgebra, we have the equalities (b3-1)-(b3-8) replacing $t_{D, D}$ for $t_{D, D}^{\prime}$ (see [2, equalities (13) to (14)]).

As an example, if $\mathcal{C}$ is symmetric and $t_{D, D}=c_{D, D}=t_{D, D}^{\prime}$ (that is, the weak YangBaxter operator is the braiding of the symmetric category $\mathcal{C}$ ) then $\nabla_{D \otimes D}=\mathrm{id}_{D \otimes D}$ and the last definition is the usual definition of weak bialgebra (see [4]).

When $\mathcal{C}$ is braided with braiding $c$ and $t_{D, D}=c_{D, D}, t_{D, D}^{\prime}=c_{D, D}^{-1}$, we have $\nabla_{D \otimes D}=\operatorname{id}_{D \otimes D}$ and $D$ is called a weak bialgebra in the braided category $\mathcal{C}$. In this setting the definition can be simplified in the following way. A weak bialgebra in a braided category $\mathcal{C}$ with braiding $c$ is an object in $\mathcal{C}$ with an algebra structure $\left(D, \eta_{D}, \mu_{D}\right)$ and a coalgebra structure $\left(D, \varepsilon_{D}, \delta_{D}\right)$ satisfying:

(c1) $\delta_{D} \circ \mu_{D}=\left(\mu_{D} \otimes \mu_{D}\right) \circ\left(D \otimes c_{D, D} \otimes D\right) \circ\left(\delta_{D} \otimes \delta_{D}\right)$;

(c2) $\varepsilon_{D} \circ \mu_{D} \circ\left(\mu_{D} \otimes D\right)=\left(\left(\varepsilon_{D} \circ \mu_{D}\right) \otimes\left(\varepsilon_{D} \circ \mu_{D}\right)\right) \circ\left(D \otimes \delta_{D} \otimes D\right)$ $=\left(\left(\varepsilon_{D} \circ \mu_{D}\right) \otimes\left(\varepsilon_{D} \circ \mu_{D}\right)\right) \circ\left(D \otimes\left(c_{D, D}^{-1} \circ \delta_{D}\right) \otimes D\right)$;

(c3) $\left(\delta_{D} \otimes D\right) \circ \delta_{D} \circ \eta_{D}=\left(D \otimes \mu_{D} \otimes D\right) \circ\left(\left(\delta_{D} \circ \eta_{D}\right) \otimes\left(\delta_{D} \circ \eta_{D}\right)\right)$ $=\left(D \otimes\left(\mu_{D} \circ c_{D, D}^{-1}\right) \otimes D\right) \circ\left(\left(\delta_{D} \circ \eta_{D}\right) \otimes\left(\delta_{D} \circ \eta_{D}\right)\right)$.

The weak bialgebra $D$ in $\mathcal{C}$ is called a weak Hopf algebra in the braided category $\mathcal{C}$ when we have the following property.

(c4) There exists a morphism $\lambda_{D}: D \rightarrow D$ in $\mathcal{C}$ (called the antipode of $D$ ) satisfying: $(\mathrm{c} 4-1) \mathrm{id}_{D} \wedge \lambda_{D}=\left(\left(\varepsilon_{D} \circ \mu_{D}\right) \otimes D\right) \circ\left(D \otimes c_{D, D}\right) \circ\left(\left(\delta_{D} \circ \eta_{D}\right) \otimes D\right)$;

$(\mathrm{c} 4-2) \lambda_{D} \wedge \mathrm{id}_{D}=\left(D \otimes\left(\varepsilon_{D} \circ \mu_{D}\right)\right) \circ\left(c_{D, D} \otimes D\right) \circ\left(D \otimes\left(\delta_{D} \circ \eta_{D}\right)\right)$; (c4-3) $\lambda_{D} \wedge \mathrm{id}_{D} \wedge \lambda_{D}=\lambda_{D}$.

Bialgebras in braided categories, in the sense of Majid [7], and classical bialgebras are examples of weak braided bialgebras in this setting. Also, braided bialgebras, in the sense of Takeuchi [11], are examples of weak braided bialgebras. Finally, recall that the notion of weak Hopf algebra in a braided category was recently called weak Hopf monoid by Pastro and Street (see [9]). In this last reference we can see that it is possible to obtain examples of weak braided Hopf algebras and weak braided bialgebras working with separable Frobenius algebras in a braided monoidal category.

Definition 2.2 can be improved using the following result.

LEMMA 2.3. Let $D$ be an object in $\mathcal{C}$ with an algebra structure $\left(D, \eta_{D}, \mu_{D}\right)$ and a coalgebra structure $\left(D, \varepsilon_{D}, \delta_{D}\right)$ such that there exists a weak Yang-Baxter operator $t_{D, D}: D \otimes D \rightarrow D \otimes D$ with associated idempotent $\nabla_{D \otimes D}$.

(1) If the equalities (b1-1) and (b3-3) of Definition 2.2 hold then (b3-1) holds.

(2) If the equalities (b1-1) and (b3-4) of Definition 2.2 hold then (b3-2) holds. 
(3) If the equalities (b2-1) and (b3-7) of Definition 2.2 hold then (b3-5) holds.

(4) If the equalities (b2-1) and (b3-8) of Definition 2.2 hold then (b3-6) holds.

PROOF. We prove assertion (1). The proofs for the assertions (2), (3) and (4) are similar and we leave the details to the reader.

Composing with $D \otimes t_{D, D}^{\prime}$ in (b3-3) and using (a2-3) and (b1-1) we have

$$
\begin{aligned}
t_{D, D} & \circ\left(\mu_{D} \otimes D\right) \circ\left(D \otimes t_{D, D}^{\prime}\right) \\
& =\left(D \otimes \mu_{D}\right) \circ\left(t_{D, D} \otimes D\right) \circ\left(D \otimes \nabla_{D \otimes D}\right) \\
& =\left(D \otimes\left(\mu_{D} \circ \nabla_{D \otimes D}\right)\right) \circ\left(t_{D, D} \otimes D\right) \\
& =\left(D \otimes \mu_{D}\right) \circ\left(t_{D, D} \otimes D\right) .
\end{aligned}
$$

Finally, composing with $\eta_{D} \otimes D \otimes \eta_{D}$ in the last equalities we obtain (b3-1).

Hence (b3-1), (b3-2), (b3-5) and (b3-6) can be removed in Definition 2.2. The preceding Lemma 2.3 implies also that in the definition of braided Hopf algebra (braided bialgebra) introduced by Takeuchi in [11] (see Definition 5.1), there exists superfluous conditions, that is, the commuting relations for the unit, the counit and the Yang-Baxter operator.

Definition 2.4. A right-right weak entwining structure in $\mathcal{C}$ is a triple $\left(A, C, \psi_{R R}\right)$ where $A$ is an algebra, $C$ is a coalgebra and $\psi_{R R}: C \otimes A \rightarrow A \otimes C$ is a morphism satisfying the following conditions, in which $e_{R R}: C \rightarrow A$ is the morphism defined by $e_{R R}=\left(A \otimes \varepsilon_{C}\right) \circ \psi_{R R} \circ\left(C \otimes \eta_{A}\right)$ :

(d1) $\psi_{R R} \circ\left(C \otimes \mu_{A}\right)=\left(\mu_{A} \otimes C\right) \circ\left(A \otimes \psi_{R R}\right) \circ\left(\psi_{R R} \otimes A\right)$;

(d2) $\psi_{R R} \circ\left(C \otimes \eta_{A}\right)=\left(e_{R R} \otimes C\right) \circ \delta_{C}$;

$(\mathrm{d} 3)\left(A \otimes \delta_{C}\right) \circ \psi_{R R}=\left(\psi_{R R} \otimes C\right) \circ\left(C \otimes \psi_{R R}\right) \circ\left(\delta_{C} \otimes A\right)$;

(d4) $\left(A \otimes \varepsilon_{C}\right) \circ \psi_{R R}=\mu_{A} \circ\left(e_{R R} \otimes A\right)$.

For a right-right weak entwining structure, by $\Delta_{R R}$ we denote the idempotent morphism

$$
\Delta_{R R}=\left(\mu_{A} \otimes C\right) \circ\left(A \otimes \psi_{R R}\right) \circ\left(A \otimes C \otimes \eta_{A}\right): A \otimes C \rightarrow A \otimes C .
$$

Similarly one can define a left-left weak entwining structure $\left(A, C, \psi_{L L}\right)$ for an algebra $A$, a coalgebra $C$ and a morphism $\psi_{L L}: A \otimes C \rightarrow C \otimes A$, that verifies similar equalities to the previous ones. Here $e_{L L}=\left(\varepsilon_{C} \otimes A\right) \circ \psi_{L L} \circ\left(\eta_{A} \otimes C\right)$ and by $\Delta_{L L}$ we denote the idempotent morphism

$$
\Delta_{L L}=\left(C \otimes \mu_{A}\right) \circ\left(\psi_{L L} \otimes A\right) \circ\left(\eta_{A} \otimes C \otimes A\right): C \otimes A \rightarrow C \otimes A .
$$

If $\left(A, C, \psi_{R R}\right)$ is a right-right entwining structure and $e_{R R}=\varepsilon_{C} \otimes \eta_{A}$ we recover the notion of right-right entwining structure or, equivalently, $-\otimes \psi_{R R}:-\otimes C \otimes$ $A \rightarrow-\otimes A \otimes C$ is a mixed distributive law (see [3]) between the monad $-\otimes A$ and the comonad $-\otimes C$. 
THEOREM 2.5. Let $D$ be an algebra-coalgebra in $\mathcal{C}$. Let $t_{D, D}: D \otimes D \rightarrow D \otimes D$ be a morphism in $\mathcal{C}$. The following assertions are equivalent.

(1) The following all hold.

(1-1) The triple $\left(D, D, \psi_{R R}=t_{D, D}\right)$ is a right-right weak entwining structure.

(1-2) The triple $\left(D, D, \psi_{L L}=t_{D, D}\right)$ is a left-left weak entwining structure.

(1-3) The morphisms $\Delta_{R R}$ and $\Delta_{L L}$ are equal.

(1-4) The morphisms $e_{R R}$ and $e_{L L}$ are equal.

(1-5) The morphisms $e_{R R}$ and $e_{L L}$ satisfy $e_{R R} \wedge \mathrm{id}_{D}=\mathrm{id}_{D} \wedge e_{L L}=\mathrm{id}_{D}$.

(2) There exists a unique idempotent morphism $\nabla_{D \otimes D}: D \otimes D \rightarrow D \otimes D$ such that

the triple $\left(D, t_{D, D}, \nabla_{D \otimes D}\right)$ satisfies the identities (b1) to (b3) of Definition 2.2.

REMARK 2.6. Note that in this theorem it is impossible to remove conditions (b3-1), (b3-2), (b3-5) and (b3-6) because $t_{D, D}$ is only a morphism and not a weak YangBaxter operator.

PROOF. First we prove that (1) implies (2). Put $\nabla_{D \otimes D}=\Delta_{R R}$. Using the fact that $\left(D, D, t_{D, D}\right)$ is a right-right and a left-left weak entwining structure we obtain (b3-3), (b3-4), (b3-7) and (b3-8). Also, we have (b3-1) because

$$
\nabla_{D \otimes D} \circ\left(\eta_{D} \otimes D\right)=\Delta_{R R} \circ\left(\eta_{D} \otimes D\right)=t_{D, D} \circ\left(D \otimes \eta_{D}\right) .
$$

The equality (b3-2) follows from

$$
\nabla_{D \otimes D} \circ\left(D \otimes \eta_{D}\right)=\Delta_{R R} \circ\left(D \otimes \eta_{D}\right)=\Delta_{L L} \circ\left(D \otimes \eta_{D}\right)=t_{D, D} \circ\left(\eta_{D} \otimes D\right),
$$

where we used that $\Delta_{R R}=\Delta_{L L}$.

As a consequence of the identity $e_{R R}=e_{L L}$ we obtain that

$$
\begin{aligned}
\nabla_{D \otimes D} & =\Delta_{R R}=\left(\left(\mu_{D} \circ\left(D \otimes e_{R R}\right)\right) \otimes D\right) \circ\left(D \otimes \delta_{D}\right) \\
& =\left(\left(\mu_{D} \circ\left(D \otimes e_{L L}\right)\right) \otimes D\right) \circ\left(D \otimes \delta_{D}\right) \\
& =\left(\left(\left(\varepsilon_{D} \otimes D\right) \circ t_{D, D}\right) \otimes D\right) \circ\left(D \otimes \delta_{D}\right),
\end{aligned}
$$

and, composing with $D \otimes \varepsilon_{D}$, we have $\left(D \otimes \varepsilon_{D}\right) \circ \nabla_{D \otimes D}=\left(\varepsilon_{D} \otimes D\right) \circ t_{D, D}$; that is, (b3-5) holds. In a similar way,

$$
\nabla_{D \otimes D}=\Delta_{L L}=\left(D \otimes\left(\left(\left(D \otimes \varepsilon_{D}\right) \circ t_{D, D}\right)\right)\right) \circ\left(\delta_{D} \otimes D\right)
$$

and then composing with $\varepsilon_{D} \otimes D$ we have (b3-6).

On the other hand, using the identity $\operatorname{id}_{D} \wedge e_{R R}=\mathrm{id}_{D}$ and the associativity of $\mu_{D}$, we obtain (b1-1) because

$$
\begin{aligned}
\mu_{D} \circ \nabla_{D \otimes D} & =\mu_{D} \circ\left(\left(\mu_{D} \circ\left(D \otimes e_{R R}\right)\right) \otimes D\right) \circ\left(D \otimes \delta_{D}\right) \\
& =\mu_{D} \circ\left(D \otimes\left(e_{R R} \wedge \mathrm{id}_{D}\right)\right)=\mu_{D} .
\end{aligned}
$$

Also, by the associativity of $\mu_{D}$ and (1-3) we have (b1-2) and (b1-3). Finally the proofs for (b2-1), (b2-2) and (b2-3) are similar and the idempotent morphism $\nabla_{D \otimes D}$ is the unique one such that $\left(D, t_{D, D}, \nabla_{D \otimes D}\right)$ satisfies the identities (b1) to (b3) of 
Definition 2.2, because if $\Omega: D \otimes D \rightarrow D \otimes D$ is another idempotent satisfying these conditions, using (b1-2) and (b3-2) we obtain

$$
\begin{aligned}
\Omega & =\Omega \circ\left(\left(\mu_{D} \circ\left(D \otimes \eta_{D}\right)\right) \otimes D\right)=\left(\mu_{D} \otimes D\right) \circ\left(D \otimes\left(\Omega \circ\left(\eta_{D} \otimes D\right)\right)\right) \\
& =\left(\mu_{D} \otimes D\right) \circ\left(D \otimes\left(t_{D, D} \circ\left(D \otimes \eta_{D}\right)\right)\right)=\Delta_{R R}=\nabla_{D \otimes D} .
\end{aligned}
$$

Conversely, suppose that there exists an unique idempotent morphism $\nabla_{D \otimes D}$ : $D \otimes D \rightarrow D \otimes D$ such that the triple $\left(D, t_{D, D}, \nabla_{D \otimes D}\right)$ satisfies the identities (b1) to (b3) of Definition 2.2. Firstly we show (1-3). Note that by (b1-2) and (b3-2) we have

$$
\begin{aligned}
\nabla_{D \otimes D} & =\nabla_{D \otimes D} \circ\left(\left(\mu_{D} \circ\left(D \otimes \eta_{D}\right)\right) \otimes D\right) \\
& =\left(\mu_{D} \otimes D\right) \circ\left(D \otimes\left(\nabla_{D \otimes D} \circ\left(\eta_{D} \otimes D\right)\right)\right) \\
& =\left(\mu_{D} \otimes D\right) \circ\left(D \otimes\left(t_{D, D} \circ\left(D \otimes \eta_{D}\right)\right)\right)=\Delta_{R R} .
\end{aligned}
$$

On the other hand, by (b1-3), (b3-1) and using a similar computations we obtain $\nabla_{D \otimes D}=\Delta_{L L}$. Note that if we work with the identities (b2-2), (b3-6), (b2-3) and (b3-5) we prove that

$$
\begin{aligned}
& \left(D \otimes\left(\left(D \otimes \varepsilon_{D}\right) \circ t_{D, D}\right)\right) \circ\left(\delta_{D} \otimes D\right) \\
& \quad=\nabla_{D \otimes D}=\left(\left(\left(\varepsilon_{D} \otimes D\right) \circ t_{D, D}\right) \otimes D\right) \circ\left(D \otimes \delta_{D}\right) .
\end{aligned}
$$

By (b3-2) and (b3-5) we have

$$
\begin{aligned}
e_{R R} & =\left(D \otimes \varepsilon_{D}\right) \circ t_{D, D} \circ\left(D \otimes \eta_{D}\right)=\left(D \otimes \varepsilon_{D}\right) \circ \nabla_{D \otimes D} \circ\left(\eta_{D} \otimes D\right) \\
& =\left(D \otimes \varepsilon_{D}\right) \circ t_{D, D} \circ\left(\eta_{D} \otimes D\right)=e_{L L} .
\end{aligned}
$$

Also, by (b3-3), (b3-4), (b3-7) and (b3-8) we have the first and the third axioms of the definitions of right-right and left-left entwining structure for $\left(D, D, t_{D, D}\right)$.

Moreover,

$$
\begin{aligned}
\mu_{D} \circ\left(e_{R R} \otimes D\right) & =\mu_{D} \circ\left(e_{L L} \otimes D\right)=\left(\varepsilon_{D} \otimes \mu_{D}\right) \circ\left(\left(t_{D, D} \circ\left(\eta_{D} \otimes D\right)\right) \otimes D\right) \\
& =\left(\varepsilon_{D} \otimes D\right) \circ \Delta_{L L}=\left(\varepsilon_{D} \otimes D\right) \circ \nabla_{D \otimes D}=\left(D \otimes \varepsilon_{D}\right) \circ t_{D, D}
\end{aligned}
$$

and

$$
\begin{aligned}
\left(e_{R R} \otimes D\right) \circ \delta_{D} & =\left(e_{L L} \otimes D\right) \circ \delta_{D}=\Delta_{L L} \circ\left(\eta_{D} \otimes D\right) \\
& =\Delta_{R R} \circ\left(\eta_{D} \otimes D\right)=t_{D, D} \circ\left(D \otimes \eta_{D}\right) .
\end{aligned}
$$

Also,

$$
\mu_{D} \circ\left(D \otimes e_{L L}\right)=\left(\varepsilon_{D} \otimes D\right) \circ t_{D, D}, \quad\left(D \otimes e_{L L}\right) \circ \delta_{D}=t_{D, D} \circ\left(\eta_{D} \otimes D\right) .
$$

Therefore $\left(D, D, t_{D, D}\right)$ is a right-right and a left-left weak entwining structure. Finally, by (b2-1) we have

$$
\begin{aligned}
e_{R R} \wedge \mathrm{id}_{D} & =e_{L L} \wedge \mathrm{id}_{D}=\left(\varepsilon_{D} \otimes \mu_{D}\right) \circ\left(t_{D, D} \otimes D\right) \circ\left(\eta_{D} \otimes \delta_{D}\right) \\
& =\left(\varepsilon_{D} \otimes D\right) \circ \nabla_{D \otimes D} \circ \delta_{D}=\left(\varepsilon_{D} \otimes D\right) \circ \delta_{D}=\mathrm{id}_{D},
\end{aligned}
$$

and by (b1-1) we prove the equality $\operatorname{id}_{D} \wedge e_{R R}=\mathrm{id}_{D}$. 
Definition 2.7. A right-left weak entwining structure on $\mathcal{C}$, consists of a triple $\left(A, C, \psi_{R L}\right)$, where $A$ is an algebra, $C$ a coalgebra, and $\psi_{R L}: A \otimes C \rightarrow A \otimes C$ a morphism such that there exist morphisms $\tau_{A, C}: A \otimes C \rightarrow C \otimes A, \sigma_{C, A}: C \otimes A \rightarrow$ $A \otimes C, r_{C, C}: C \otimes C \rightarrow C \otimes C$ and $s_{C, C}: C \otimes C \rightarrow C \otimes C$ satisfying the following relations, in which $e_{R L}: C \rightarrow A$ is the morphism defined by $e_{R L}=\left(A \otimes \varepsilon_{C}\right) \circ \psi_{R L} \circ$ $\left(\eta_{A} \otimes C\right)$ :

(e1) $\left(\mu_{A} \otimes C\right) \circ\left(A \otimes \sigma_{C, A}\right) \circ\left(\psi_{R L} \otimes A\right) \circ\left(A \otimes\left(\tau_{A, C} \circ \psi_{R L}\right)\right)$ $=\psi_{R L} \circ\left(\mu_{A} \otimes C\right)$

(e2) $\left(\psi_{R L} \otimes C\right) \circ\left(A \otimes s_{C, C}\right) \circ\left(\psi_{R L} \otimes C\right) \circ\left(A \otimes\left(r_{C, C} \circ \delta_{C}\right)\right)$ $=\left(A \otimes \delta_{C}\right) \circ \psi_{R L}$

(e3) $\psi_{R L} \circ\left(\eta_{A} \otimes C\right)=\left(e_{R L} \otimes C\right) \circ \delta_{C}$;

(e4) $\left(A \otimes \varepsilon_{C}\right) \circ \psi_{R L}=\mu_{A} \circ\left(A \otimes e_{R L}\right)$.

In a similar way a left-right weak entwining structure consists of a triple $\left(A, C, \psi_{L R}\right)$, where $A$ is an algebra, $C$ a coalgebra, and $\psi_{L R}: C \otimes A \rightarrow C \otimes A$ a morphism such that there exist morphisms $\tau_{A, C}: A \otimes C \rightarrow C \otimes A, \sigma_{C, A}: C \otimes A \rightarrow$ $A \otimes C, r_{A, A}: A \otimes A \rightarrow A \otimes A$ and $s_{A, A}: A \otimes A \rightarrow A \otimes A$, satisfying the following relations, in which $e_{L R}: C \rightarrow A$ is the morphism defined by $e_{L R}=\left(\varepsilon_{C} \otimes A\right) \circ \psi_{L R} \circ$ $\left(C \otimes \eta_{A}\right)$ :

(f1) $\left(C \otimes\left(\mu_{A} \circ r_{A, A}\right)\right) \circ\left(\psi_{L R} \otimes A\right) \circ\left(C \otimes s_{A, A}\right) \circ\left(\psi_{L R} \otimes A\right)$ $=\psi_{L R} \circ\left(C \otimes \mu_{A}\right)$

(f2) $\left(C \otimes\left(\psi_{L R} \circ \tau_{A, C}\right)\right) \circ\left(\psi_{L R} \otimes C\right) \circ\left(C \otimes \sigma_{C, A}\right) \circ\left(\delta_{C} \otimes A\right)$ $=\left(\delta_{C} \otimes A\right) \circ \psi_{L R}$

(f3) $\psi_{L R} \circ\left(C \otimes \eta_{A}\right)=\left(C \otimes e_{L R}\right) \circ \delta_{C}$;

(f4) $\left(\varepsilon_{C} \otimes A\right) \circ \psi_{L R}=\mu_{A} \circ\left(e_{L R} \otimes A\right)$.

PROPOSITION 2.8. Let $D$ be a weak braided bialgebra in $\mathcal{C}$ with weak Yang-Baxter operator $t_{D, D}$ and associated idempotent $\nabla_{D \otimes D}$. Then it follows that:

(1) $\left(D, D, \psi_{1}=\left(D \otimes \mu_{D}\right) \circ\left(t_{D, D} \otimes D\right) \circ\left(D \otimes \delta_{D}\right)\right)$ is a right-right weak entwining structure;

(2) $\left(D, D, \psi_{2}=\left(\mu_{D} \otimes D\right) \circ\left(D \otimes t_{D, D}\right) \circ\left(\delta_{D} \otimes D\right)\right)$ is a left-left weak entwining structure;

(3) $\left(D, D, \psi_{3}=\left(D \otimes \mu_{D}\right) \circ\left(\delta_{D} \otimes D\right)\right)$ is a right-left weak entwining structure where $\tau_{D, D}=r_{D, D}=t_{D, D}^{\prime}$ and $\sigma_{D, D}=s_{D, D}=t_{D, D}$;

(4) $\left(D, D, \psi_{4}=\left(\mu_{D} \otimes D\right) \circ\left(D \otimes \delta_{D}\right)\right)$ is a left-right weak entwining structure where $\tau_{D, D}=r_{D, D}=t_{D, D}^{\prime}$ and $\sigma_{D, D}=s_{D, D}=t_{D, D}$.

PROOF. The assertion (1) was proved in [2, Proposition 3.2]. The proof for the other claims are similar and the detailed verification of (2), (3) and (4) is an exercise that we leave to the reader.

REMARK 2.9. For the entwining structures defined in Proposition 2.8 we denote by $e_{1}, e_{2}, e_{3}$ and $e_{4}$ the morphisms 


$$
\begin{array}{ll}
e_{1}=\left(D \otimes \varepsilon_{D}\right) \circ \psi_{1} \circ\left(D \otimes \eta_{D}\right), & e_{2}=\left(\varepsilon_{D} \otimes D\right) \circ \psi_{2} \circ\left(\eta_{D} \otimes D\right), \\
e_{3}=\left(D \otimes \varepsilon_{D}\right) \circ \psi_{3} \circ\left(\eta_{D} \otimes D\right), & e_{4}=\left(\varepsilon_{D} \otimes D\right) \circ \psi_{4} \circ\left(D \otimes \eta_{D}\right) .
\end{array}
$$

Note that $e_{1}=\Pi_{D}^{R}, e_{2}=\Pi_{D}^{L}, e_{3}=\bar{\Pi}_{D}^{L}$ and $e_{4}=\bar{\Pi}_{D}^{R}$, where $\Pi_{D}^{R}, \Pi_{D}^{L}, \bar{\Pi}_{D}^{L}$ and $\bar{\Pi}_{D}^{R}$ are the morphisms defined in Propositions 2.3, 2.4, 2.5 and 2.6 respectively of [2]. In these results we assume that $D$ is an algebra-coalgebra and $t_{D, D}$ is a weak YangBaxter operator with associated idempotent satisfying (b1), (b2), (b3) and (b4) of Definition 2.2. Then, under these conditions we have the following results.

(1) The following assertions are equivalent [2, Proposition 2.3].

(1-1) The equality $\varepsilon_{D} \circ \mu_{D} \circ\left(\mu_{D} \otimes D\right)=\left(\left(\varepsilon_{D} \circ \mu_{D}\right) \otimes\left(\varepsilon_{D} \circ \mu_{D}\right)\right) \circ(D \otimes$ $\left.\left(t_{D, D}^{\prime} \circ \delta_{D}\right) \otimes D\right)$ holds.

(1-2) The equality $\mu_{D} \circ\left(D \otimes e_{2}\right)=\left(\varepsilon_{D} \otimes D\right) \circ \psi_{2}$ holds.

(1-3) The equality $\mu_{D} \circ\left(e_{1} \otimes D\right)=\left(D \otimes \varepsilon_{D}\right) \circ \psi_{1}$ holds.

(2) The following assertions are equivalent [2, Proposition 2.4].

(2-1) The equality $\varepsilon_{D} \circ \mu_{D} \circ\left(\mu_{D} \otimes D\right)=\left(\left(\varepsilon_{D} \circ \mu_{D}\right) \otimes\left(\varepsilon_{D} \circ \mu_{D}\right)\right) \circ(D \otimes$ $\left.\delta_{D} \otimes D\right)$ holds.

(2-2) The equality $\mu_{D} \circ\left(D \otimes e_{3}\right)=\left(D \otimes \varepsilon_{D}\right) \circ \psi_{3}$ holds.

(2-3) The equality $\mu_{D} \circ\left(e_{4} \otimes D\right)=\left(\varepsilon_{D} \otimes D\right) \circ \psi_{4}$ holds.

(3) The following assertions are equivalent [2, Proposition 2.5].

(3-1) The equality $\left(\delta_{D} \otimes D\right) \circ \delta_{D} \circ \eta_{D}=\left(D \otimes\left(\mu_{D} \circ t_{D, D}^{\prime}\right) \otimes D\right) \circ\left(\left(\delta_{D} \circ \eta_{D}\right)\right.$ $\left.\otimes\left(\delta_{D} \circ \eta_{D}\right)\right)$ holds.

(3-2) The equality $\left(D \otimes e_{2}\right) \circ \delta_{D}=\psi_{2} \circ\left(\eta_{D} \otimes D\right)$ holds.

(3-3) The equality $\left(e_{1} \otimes D\right) \circ \delta_{D}=\psi_{1} \circ\left(D \otimes \eta_{D}\right)$ holds.

(4) The following assertions are equivalent [2, Proposition 2.6].

(4-1) The equality $\left(\delta_{D} \otimes D\right) \circ \delta_{D} \circ \eta_{D}=\left(D \otimes \mu_{D} \otimes D\right) \circ\left(\left(\delta_{D} \circ \eta_{D}\right) \otimes\left(\delta_{D} \circ\right.\right.$ $\left.\left.\eta_{D}\right)\right)$ holds.

(4-2) The equality $\left(e_{3} \otimes D\right) \circ \delta_{D}=\psi_{3} \circ\left(\eta_{D} \otimes D\right)$ holds.

(4-3) The equality $\left(D \otimes e_{4}\right) \circ \delta_{D}=\psi_{4} \circ\left(\eta_{D} \otimes D\right)$ holds.

From Theorem 2.5, Proposition 2.8 and Remark 2.9 we conclude the main theorem of this paper. Note that Theorem 2.10 implies that the condition $\delta_{D} \circ \mu_{D}=\left(\mu_{D} \otimes\right.$ $\left.\mu_{D}\right) \circ\left(D \otimes t_{D, D} \otimes D\right) \circ\left(\delta_{D} \otimes \delta_{D}\right)$ assumed by Caenepeel and De Groot in [5, Theorem 4.7] is superfluous (in [5], $\mathcal{C}=k$-Mod is the category of modules over a commutative ring $k$ and $t_{D, D}$ is the natural isomorphism of symmetry). Also, note that the conditions of the following theorem in the case $\mathcal{C}=k$-Mod are trivial because $t_{D, D}$ is the natural isomorphism of symmetry.

Theorem 2.10. Let $D$ be an algebra-coalgebra in $\mathcal{C}$. Let $t_{D, D}$ be a weak YangBaxter operator with associated idempotent $\nabla_{D \otimes D}$ such that $\left(D, D, t_{D, D}\right)$ is a rightright and a left-left weak entwining structure, $\nabla_{D \otimes D}=\Delta_{R R}=\Delta_{L L}, e_{R R}=e_{L L}$, and $e_{R R} \wedge \mathrm{id}_{D}=\mathrm{id}_{D} \wedge e_{R R}=\mathrm{id}_{D}$. The following assertions are equivalent:

(1) $D$ is a weak braided bialgebra;

(2) ( $\left.D, D, \psi_{1}\right)$ is a right-right weak entwining structure and $\left(D, D, \psi_{3}\right)$ is a rightleft weak entwining structure; 
(3) (D, D, $\left.\psi_{2}\right)$ is a left-left weak entwining structure and $\left(D, D, \psi_{4}\right)$ is a left-right weak entwining structure;

(4) $\left(D, D, \psi_{2}\right)$ is a left-left weak entwining structure and $\left(D, D, \psi_{3}\right)$ is a right-left weak entwining structure;

(5) $\left(D, D, \psi_{1}\right)$ is a right-right weak entwining structure and $\left(D, D, \psi_{4}\right)$ is a leftright weak entwining structure.

PROOF. We begin the proof emphasizing that by Theorem 2.5 the equalities contained in (b1), (b2) and (b3) of Definition 2.2 hold. Then if (b4) holds we can apply [2, Propositions 2.3, 2.4, 2.5, and 2.6].

Firstly, by (1) of Proposition 2.8 we have (1) implies (2). To prove (2) implies (1), note that we have (b4) because $\left(D, D, \psi_{1}\right)$ is a right-right entwining structure. Indeed,

$$
\begin{aligned}
\delta_{D} \circ \mu_{D} & =\nabla_{D \otimes D} \circ \delta_{D} \circ \mu_{D}=\Delta_{L L} \circ \delta_{D} \circ \mu_{D} \\
& =\left(D \otimes \mu_{D}\right) \circ\left(t_{D, D} \otimes D\right) \circ\left(\eta_{D} \otimes\left(\delta_{D} \circ \mu_{D}\right)\right)=\psi_{1} \circ\left(\eta_{D} \otimes \mu_{D}\right) \\
& =\left(\mu_{D} \otimes D\right) \circ\left(D \otimes \psi_{1}\right) \circ\left(\psi_{1} \otimes D\right) \circ\left(\eta_{D} \otimes D \otimes D\right) \\
& =\left(\mu_{D} \otimes \mu_{D}\right) \circ\left(D \otimes t_{D, D} \otimes D\right) \circ\left(\left(\nabla_{D \otimes D} \circ \delta_{D}\right) \otimes \delta_{D}\right) \\
& =\left(\mu_{D} \otimes \mu_{D}\right) \circ\left(D \otimes t_{D, D} \otimes D\right) \circ\left(\delta_{D} \otimes \delta_{D}\right) .
\end{aligned}
$$

Then, using the fact that $\mu_{D} \circ\left(D \otimes e_{3}\right)=\left(D \otimes \varepsilon_{D}\right) \circ \psi_{3}$ and $\mu_{D} \circ\left(e_{1} \otimes D\right)=$ $\left(D \otimes \varepsilon_{D}\right) \circ \psi_{1}$ hold, by [2, Propositions 2.4 and 2.3] we obtain (b5) of Definition 2.2. Also, using the fact that $\left(e_{1} \otimes D\right) \circ \delta_{D}=\psi_{1} \circ\left(D \otimes \eta_{D}\right)$ and $\left(e_{3} \otimes D\right) \circ \delta_{D}=\psi_{3} \circ$ $\left(\eta_{D} \otimes D\right)$ hold we have (b6).

Finally, repeating similar arguments it is possible to prove (1) if and only if (3), (1) if and only if (4), (1) if and only if (5) and the proof is finished.

\section{References}

[1] J. N. Alonso Álvarez, J. M. Fernández Vilaboa and R. González Rodríguez, 'Weak Hopf algebras and weak Yang-Baxter operators', J. Algebra 320 (2008), 2101-2143.

[2] J. N. Alonso Ấlvarez, J. M. Fernández Vilaboa and R. González Rodríguez, 'Weak braided Hopf algebras', Indiana Univ. Math. J. 57 (2008), 2423-2458.

[3] J. Beck, 'Distributive laws', in: Seminar on Triples and Categorical Homology Theory, Lecture Notes in Mathematics, 80 (ed. B. Eckmann) (Springer, Berlin, 1969), pp. 119-140.

[4] G. Böhm, F. Nill and K. Szlachányi, 'Weak Hopf algebras, I. Integral theory and $C^{*}$-structure', J. Algebra 221 (1999), 385-438.

[5] S. Caenepeel and E. De Groot, 'Modules over weak entwining structures', in: New Trends in Hopf Algebra Theory, Contemporary Mathematics, 267 (American Mathematical Society, Providence, RI, 2000), pp. 31-54.

[6] A. Joyal and R. Street, 'Braided tensor categories', Adv. Math. 102 (1993), 20-78.

[7] S. Majid, 'Algebras and Hopf algebras in braided categories', in: Advances in Hopf Algebras, Lecture Notes in Pure and Applied Mathematics, 158 (Marcel Dekker, New York, 1994), pp. 55-105.

[8] S. Majid, 'Cross products by braided groups and bosonization', J. Algebra 163 (1994), 165-190.

[9] C. Pastro and R. Street, 'Weak Hopf monoids in braided monoidal categories', Algebra and Number Theory, at press (2008). 
[10] D. E. Radford, 'The structure of Hopf algebras with a projection', J. Algebra 92 (1985), 322-347.

[11] M. Takeuchi, 'Survey of braided Hopf algebras', in: New Trends in Hopf Algebra Theory, Contemporary Mathematics, 267 (American Mathematical Society, Providence, RI, 2000), pp. 301-323.

J. N. ALONSO ÁLVAREZ, Departamento de Matemáticas, Universidad de Vigo, Campus Universitario Lagoas-Marcosende, E-36280 Vigo, Spain

e-mail: jnalonso@uvigo.es

J. M. FERNÁNDEZ VILABOA, Departamento de Álxebra, Universidad de Santiago de Compostela, E-15771 Santiago de Compostela, Spain

e-mail: alvila@usc.es

R. GONZÁLEZ RODRÍGUEZ, Departamento de Matemática Aplicada II, Universidad de Vigo, Campus Universitario Lagoas-Marcosende, E-36310 Vigo, Spain

e-mail:rgon@dma.uvigo.es 